\title{
Transdermal Drug Delivery of Asiatic Acid Influences Renal Function and Electrolyte Handling in Plasmodium berghei - Infected Sprague-Dawley Male Rats
}

\author{
Mavondo Greanious Alfred ${ }^{1,2,{ }^{*}, \text { Musabayane Cephas Tagumirwa }}{ }^{2}$ \\ ${ }^{1}$ College of Health Sciences, University of Kwa Zulu Natal, Durban, South Africa \\ ${ }^{2}$ Pathology Department, National University of Science and Technology (NUST), Bulawayo, Zimbabwe
}

Email address:

greaniousa@gmail.com(M. G.Alfred), greanious.mavondo@nust.ac.zw (M. G.Alfred)

${ }^{*}$ Corresponding author

\section{To cite this article:}

Mavondo Greanious Alfred, Musabayane Cephas Tagumirwa. Transdermal Drug Delivery of Asiatic Acid Influences Renal Function and Electrolyte Handling in Plasmodium berghei - Infected Sprague-Dawley Male Rats. Journal of Diseases and Medicinal Plants. Vol. 4, No. 1, 2017, pp. 18-29. doi: 10.11648/j.jdmp.20180401.13

Received: October 24, 2017; Accepted: November 20, 2017; Published: April 3, 2018

\begin{abstract}
Background: Malaria is one of the most important infectious inflammatory diseases worldwide which affects vital organs including gastrointestinal and kidneys. Higher prevalence of malaria related renal failure, current malaria drugs nephrotoxicity and drug resistance to malaria calls for continued research in anti-parasitic as well as anti-disease inflammatory pharmaceuticals. Asiatic acid, a plant phytochemical extract, has antioxidant, pro-oxidant and diuretic properties. Here we report influence of Asiatic acid-pectin hydrogel matrix patch application in P. berghei-infected Sprague Dawley rats on renal function and electrolyte handling. Materials and Methods: Asiatic acid $(5 \mathrm{mg} / \mathrm{kg})$-pectin patch was applied on the dorsal neck region of the rat on day 7 post infection and monitored for parasitaemia, and physicochemical changes. Urine and blood were collected for measuring various biochemical parameters to estimate renal function and electrolyte handling. Results: Asiatic acid-pectin patch application had significant influence on food and water intake as well as weight changes, urine electrolytes, glomerular filtration rate, and antioxidant markers together with hormonal changes of aldosterone and vasopressin. Conclusion: Application of the once-off Asiatic acid $(5 \mathrm{mg} / \mathrm{kg})$-pectin patch influence renal function and renal electrolyte handling while ameliorating, biochemical and hormonal derangements induced by malaria. Novelty of the Work Here we show for the first time a) the efficacy of Asiatic acid (AA) in suppressing murine malaria by way of administering the phytochemical using the amidated pectin hydrogel matrix patch transdermal drug delivery system, b) diminution of asiatic acid dose applied from $10 \mathrm{mg} / \mathrm{kg}$ oral to $5 \mathrm{mg} / \mathrm{kg}$ by transdermal, c) reduction of time once-off patch application from five days to three days, d) attenuation of oxidative and hormonal derangements in malaria and e) the amelioration of renal function together with improvement in renal electrolyte handling. The results may be of benefit in patient care replacing the multiple dosing approaches used in malaria management.
\end{abstract}

Keywords: Asiatic Acid, Malaria, Transdermal Drug Delivery, Plasmodium berghei, Sprague Dawley Rats, Renal Failure

\section{Introduction}

Malaria has remained one of the most recalcitrant disease with its varied sequalae that imamate from the bite of the anopheles mosquito during its feeding on infected human blood meal when the Plasmodium parasite is transmitted to human hosts. The parasite is an obligate intracellular protozoa invading several body systems and organs during the infection. Different developmental stages of the parasite has been demonstrated to be present in the intravascular compartment (red blood cells-RBC's), hepatocytes, gastrointestinal cells, the renal system and the brain tissue which are invaded causing different degrees of damage in each organ [1]. Gut and hepatic invasion by the parasite leads to inflammatory gut and hepatic syndromes. Consequently, malaria present a number of fatalistic syndromes dependant 
on the organ system mostly affected although the symptomatic expression of the disease is mainly associated with the blood stage of the parasites. The malarial triad include fever, anaemia, and splenomegaly. However, gastrointestinal symptoms, such as abdominal pain, diarrhoea, and vomiting, develop in patients with falciparum malaria [39, 40]. Small intestine pathological changes of tongue-shaped jejunum and duodenum villi [34], width extended and shortened villi [13], villi rapture and bleeding contribute to gastrointestinal malfunction $[6,45,48]$ leading to fluid loss in malaria compromising renal function. Moreover, the anorexic phenotype which causes malnutrition and the associated fluid losses through increased intestinal permeability in malarial [49] leads to several changes that affect and dysregulate the gut-brain axis leading to dehydration, hypovolaemia, renal insufficiency, cachexia and death. The intestinal bleeding induced may hypovolaemia and bleeding may leads to an oxidative propensity through release of free haemoglobin and high grade inflammation by way of immune system activation with resultant renal electrolyte handling dysregulation, acute kidney injury and renal dysfunction amongst other derangements [2].

In malaria, acute renal failure (ARF) may develop without warning when the disease is not properly managed. Malaria ARF (MARF) may develop into malaria chronic renal failure (MCRF) or the later might develop independently without an acute phase [17]. In non-immune adults and children MARF is one of the leading causes of ARF in Africa South of the Sahara [32]. Mortality from MARF has been rated at $14-45 \%$ [3]. Aetiology of MARF depends on the immunological and inflammatory host responses [37], microcirculation blockage by non-parasitized red blood (npRBC's) cells and parasitized red blood cells [pRBC's] [11, 25], hypovolaemia from peripheral blood pooling and ischaemic acute tubular necrosis [ATN] [33]. Treatment, therefore should be aimed at avoiding ARF development or reversing the renal pathophysiology where it has occurred.

Current malaria treatment regimens have a number of setbacks. Amongst these, use of 4-or 8-aminoquinolene drugs in malaria has been associated with increased sodium wasting and hyperkalaemia [31]. Artemisinin and it derivatives, current drugs of choice in malaria treatment, rapidly clear parasitaemia but create reactive oxygen species (ROS) leading to red blood cells (RBC's) damage with post artemisinin treatment induced drug haemolysis (PADH) and ARF [16, 38, 42]. Moreover, multi-drug resistance has emerged in antimalarial treatment regimens. This introduces one of the most prominent obstacle in the fight against malaria. Therefore, continuing antimalarial research is paramount especially one that is aimed at amelioration of malaria pathophysiology like MARF.

Plant extracts including phytochemicals open up an avenue worth exploring as anti-parasitaemia as well as malaria renal pathophysiology (anti-disease) alexia. Per oral administration of Asiatic acid (AA) has been demonstrated to have both anti-parasitic as well as anti-disease influence in malarial complications. This followed pre-infection administration of
AA (chemoprophylaxis) and post-infection administration of the phytochemical in Plasmodium berghei-infected male Sprague Dawley rats [20]. Also worth noting in these studies is the observation that AA $(10 \mathrm{mg} / \mathrm{kg}$, as an oral concoction, was able to suppress parasitaemia in Plasmodium bergheiinfected Sprague Dawley rats as well as amelioration of severe malarial anaemia [22]. Furthermore, an even lower dose of AA as an AA $(5 \mathrm{mg} / \mathrm{kg})$-amidated pectin hydrogel patch was shown to suppress parasitaemia as well as to ameliorate severe malarial anaemia (SMA) in a postinfection treatment experimentation [23]. Not only does this approach increase the number of potential antimalarial therapies, but it also introduces a possible pathophysiologydirected approach for both malaria prevention and treatment.

Asiatic acid is both an antioxidant (hydrogen bond acceptor- BDA) and a pro-oxidant (hydrogen-bond donorHBD) capable of redox-reactions participation [36] with a potential to eradicate the parasite while ameliorating untoward malaria disease sequelae. AA is pleiotropic in nature $[12,50,51]$ with its diuretic effect [35] and nonnephrotoxicity [52] makes it more amenable in the management of MARF. However, the influence of AA in the management of MARF has not been reported. Interestingly, it makes sense that therapeutics with anti- inflammatory targets in malaria have a higher success chance in eradicating the parasite as well as forefending MARF development.

Renal dysfunction in malaria is commonly associated with electrolytes mismanagement by the nephron accompanied by arginine vasopressin (AVP) and aldosterone (ALD) aberrations before overt disease signs and symptoms are apparent $[30,31]$ through increased oxidative stress from oxygen free radicals and $\mathrm{ONOO}^{-}[7,18]$. The hormonal and electrolyte abnormalities formulate the renal disease aspects of malaria, as contrasted to parasitic infection, which need to be managed concurrently with parasitaemia reduction strategies. Hypovolaemia of malaria, through red blood cell (RBC's) haemolysis and reduced water and food intake, increases both AVP and ALD. Therefore, investigation of MARF will, of necessity, involve demonstration of both AVP and ALD activities together with changes in electrolytes concentrations in both plasma and urine. The influence of AA administration on AVP and ALD in malaria, renal function or any other disease is not yet reported. Also still a grey area exists to AA's antioxidant and anti-inflammatory capacities in malarial electrolyte handling.

Studies have indicated that the mode of drug delivery, e. g. transdermal drug delivery system (TDDS) compared to oral administration, plays a crucial role in the ultimate efficacies of the drug to the extent of salvaging antimalarial potency of chloroquine [26, 28]. As a result to further enhance the efficacy and reduce the dosage of AA in malaria management, an AA-amidated pectin hydrogel matrix patch, commonly referred to as "the patch" was mooted as a novel method of phytochemical drug delivery and antimalarial therapy. Indeed, transdermal delivery of AA has been reported to result in the reduction of the dose administered and in suppressing parasitaemia [23]. We hypothesised that 
the antioxidant capacity of AA may influence electrolyte transporters by reducing oxidative stress, which is common in malaria infection, and thus play a crucial antiinflammatory role in malaria pathology.

Therefore, as a follow up, the aim of this study was to investigate the influence of AA-TDDS on malarial progression, malaria nutritional status, malaria antioxidant capacity, host renal function and renal electrolyte handling in male Sprague Dawley rats as a novel approach in both AA administration and AA malaria treatment.

\section{Materials and Methods}

\subsection{Materials}

\subsubsection{Drugs, Chemical and Accessories}

Asiatic acid (AA $>97 \%$ purity), Giemsa stain, dimethyl sulphoxide (DMSO), chloroquine diphosphate were purchased from Sigma-Aldrich (St. Louis, Missouri, USA). All other chemicals and reagents used were obtained from reputable suppliers and were of analytical grade.

\subsubsection{Animals}

Male Sprague-Dawley (SD) rats weighing 90-120 g (6 weeks old) were obtained from Biomedical Research Unit (BRU), of the University of KwaZulu-Natal (UKZN) where they were bred and housed for the entire experimental period. The animals were kept under maintained laboratory conditions of constant temperature $\left(22 \pm 1^{\circ} \mathrm{C}\right) ; \mathrm{CO}_{2}(<5000$ $\mathrm{ppm})$, humidity of $55 \pm 5 \%$ and illumination (12 h light/dark cycles). The animals had full access to food, standard rat chow (Meadows Feeds, Pietermaritzburg, South Africa) and water ad libitum. Infected non-treated control (IC) animals were euthanatized on day 12 post infection. All other animal groups were euthanatized sacrificed by day 21. Lethal inhalation anaesthesia with isoform (Safeline Pharmaceuticals, Rooderport, South Africa) for 3 minutes via a gas anaesthetic chamber $(100 \mathrm{mg} / \mathrm{kg})$ was used to overdose euthanatize all the animals. All experiments and protocols used in this study were reviewed and approved by the animal ethics committee of the UKZN with ethical clearance numbers 079/14/Animal and 013/15/Animal issued.

\subsubsection{Malaria Parasites}

Chloroquine-susceptible strain of Plasmodium berghei ANKA, was a kind donation from Professor Peter Smith (University of Cape Town, Division of Clinical Pharmacology, South Africa). P. berghei $\left(10^{5}\right.$ parasitized red blood cells [pRBC's] suspension in saline) was inoculated intraperitoneal (ip) [9] into stock animals which were euthanatized after 12 days and the infected blood was harvested, washed and stored at $-80^{\circ} \mathrm{C}$ in freezing media (30\% glycerol in phosphate buffer) until used.

\subsubsection{Experimental Design}

The study was a 21 day protocol of animals groups $(n=6$ per group) administered either with AA-pectin matrix patch or chloroquine-pectin patch post-infection with $P$. berghei.
The groups were as follows:

i) Non-infected treated control DMSO plus AA5 (NIC)

ii) Infected non-treated control (IC)

iii) Infected groups treated with CHQ $30 \mathrm{mg} / \mathrm{kg}$ patch (30CHQ)

iv) Infected groups treated with AA $5 \mathrm{mg} / \mathrm{kg}$ patch (AA5)

v) Non-infected administered DMSO patch absolute control (AC)

Preliminary findings have shown that AA $5 \mathrm{mg} / \mathrm{kg}$ had the highest efficacy and it will be the only dose reported here unless where it is applicable to stress a point. The AC and NIC groups did not show any difference. As a result only the NIC results are reported.

\subsection{Methods}

\subsubsection{Induction of Parasitaemia}

Chloroquine-susceptible strain of $P$. berghei ANKA $\left(10^{5}\right.$ pRBC's suspension in saline) was inoculated intraperitoneal [9] in the positive and test animals. Non infected Control Animals (NIC): Equal volume of saline was administered to the control animals.

\subsubsection{Influence of AA on Sub-Chronic Studies}

Experiments were conducted over a period of 21 days to establish the influence of $\mathrm{AA}$ on renal function and electrolyte handling in murine-malaria affected SD rats. Animals per group ( $\mathrm{n}=6$ per group) were housed individually in Makrolon polycarbonate metabolic cages (Techniplast, Labotec, South Africa) with food and water availed ad libitum.

\subsubsection{Preparation of AA-Pectin and CHQ Hydrogel Patches}

Amidated pectin hydrogel matrix patches were prepared using a previously described protocol by Musabayane et al., (2003) with slight modifications [27]. Low methoxyl amidated pectin with a degree of esterification (DE) of $23 \%$ and an amidation of $24 \%$ was used for the preparation of the AA and CHQ patches. AA $(5 \mathrm{mg} / \mathrm{kg})$ or CHQ $(30 \mathrm{mg} / \mathrm{kg})$ were added to amidated low methoxyl pectin $(4.4 \mathrm{~g})$ and dissolved in deionized water $(110 \mathrm{~mL})$ in separate beakers. The concoctions were agitated at $37^{\circ} \mathrm{C}$ in a water bath at a speed of $38 \times \mathrm{G}$ using an electric motor mixing rotor (Heidolph instruments GmbH \& Co. KG, Schwabach, Germany) for 15 minutes. Subsequently, DMSO $(2 \mathrm{~mL})$, $1.65 \mathrm{~mL}$ eucalyptus oil (Barrs Pharmaceutical Industries, Cape Town, South Africa) and $1.65 \mathrm{~mL}$ vitamin E (Pharmacare Ltd, Johannesburg, South Africa) were added to the mixtures and mixed for $1 \mathrm{~h} 30$ minutes. Aliquots $(11 \mathrm{~mL})$ were transferred to petri dishes with a known diameter and frozen at $-4^{\circ} \mathrm{C}$ for 18 hours following which $2 \% \mathrm{CaCl}_{2}$ solution was added on top of the frozen pectin and left to stand at room temperature $\left( \pm 25^{\circ} \mathrm{C}\right)$ for 10 minutes to allow for cross-linking and formation of the matrix patch. The patches were then stored in a refrigerator at $4^{\circ} \mathrm{C}$ until use.

\subsubsection{Application of the Patch}

Three discs $\left(4 \pm 1 \mathrm{~mm}^{2}\right)$ were punched out from AA 
$(5 \mathrm{mg} / \mathrm{kg})$ patch or CHQ $(30 \mathrm{mg} / \mathrm{kg})$ and applied once-off onto the shaved dorsal region of the animal from Day 7 to Day 10 (three days). The jacket holding the patch in place was made from clinically sterile adhesive fabric plaster (Mediplast, Neomedic, Rickmansworth, and Herefordshire, UK) which caused no discomfort to the animals. Notes on patch application: The dorsal neck region was selected because it was the least accessible to the animals' grooming habits and avoided removal of the patch by the animals. The transdermal delivery (TTD) system aimed at reducing phytochemical amount delivered to the animal, dosing frequency, treatment duration and general animal discomfiture. A theoretical total AA yield of the patch was estimated at $1 \mu \mathrm{g} /$ per disc for $5 \mathrm{mg} / \mathrm{kg}$ AA dose and $3 \mu \mathrm{g}$ was administered as three discs were applied per animal to provide equivalent of five day treatment.

\subsubsection{AA5-Pectin Patch Influence on Parasitaemia Monitoring}

Parasitaemia\% monitored 72 hours post inoculation (prepatent period), every third day up to day 7 [patent period] [4], every day during patent/treatment period of five days and thereafter every other day (post-treatment period) until day 21. A $15-20 \%$ parasitaemia on day 7 , confirmed by Giemsa staining under a microscope (Olympus Cooperation, Tokyo, Japan) [20], was considered as stable state malaria where administration of AA and treatment with 30CHQ was commenced

\subsubsection{AA5-Pectin Patch Influence on Physicochemical Monitoring}

Body weights, food and water intake were monitored gravimetrically using a balance Mettler balance PC 180instruments (Protea Laboratory Services, Johannesburg, South Africa) in control and treated animals at 09h00 every 3rd day during the pre-treatment, treatment and post treatment periods.

\subsubsection{Plasma and Tissue Sample Harvesting}

All animals were euthanatized by exposing to isofor (Safeline Pharmaceuticals, Rooderport, South Africa) for 3 minutes via a gas anaesthetic chamber $(100 \mathrm{mg} / \mathrm{kg})$. Blood samples were collected by cardiac puncture into pre-cooled lithium heparin tubes onto melting ice. Blood in heparin tubes was immediately centrifuged for 15 minutes at $959 \times \mathrm{G}$ in a $4^{\circ} \mathrm{C}$ centrifuge (Eppendorf International, Hamburg, Germany) and plasma separated. The liver, kidney, muscle and heart were removed, snap frozen in liquid nitrogen and stored together with the plasma in a Bio Ultra freezer (Snijers Scientific, Tilburg, Netherlands) at $-80^{\circ} \mathrm{C}$ until use. Protein content of all organs was quantified using the Lowry method and samples standardized to one concentration $(1 \mathrm{mg} / \mathrm{mL})$.

\subsubsection{AA5-Pectin Patch Influence on Urinalysis}

Urine was collected over a 24 hour period in metabolic cages every third day during the pre-patent, patent/treatment and post treatment periods to investigate influence of AA on urine parameters. Gravimetric method was used to determine urine volume. Quantitative measurements of total urinary outputs and plasma concentrations of $\mathrm{Na}^{+}, \mathrm{K}^{+}, \mathrm{Cl}^{-}$, urea and creatinine were performed using Beckman Coulter (Synchron CX3 Clinical Systems, Fullerton, California, USA) with reagent kits from Beckman Coulter (Dublin, Ireland). Creatinine concentrations used to calculate the glomerular filtration rate $(\mathrm{GFR})$ using formulare: $\mathrm{GFR}=\left(\left[\mathrm{U}_{\text {creat }}\right] \times \mathrm{UV}\right) /$ ( $\left[\mathrm{P}_{\text {creat }}\right] \times 1440$ minutes) where $\mathrm{U}_{\text {creat }}$ is urine creatinine concentration, $\mathrm{P}_{\text {creat }}$ is plasma creatinine concentration and $\mathrm{UV}$ is 24 hour urine volume. Renal clearance (C) and fractional excretions (FE) were calculated with the standard formulae $\mathrm{C}=\mathrm{U} \times \mathrm{V} / \mathrm{P}$ and $\mathrm{FE}=\mathrm{C} / \mathrm{GFR}$ where $\mathrm{U}$ is the urinary concentration, $\mathrm{V}$ is the urine flow rate and $\mathrm{P}$ is plasma concentration as used by Salman et al [43]. AA5pectin patch influence on haemolysis: To corroborate both haematological aberrations in malaria and hypoxia-induced tissue damage, plasma enzymatic activity of lactate dehydrogenase (LDH) was estimated. LDH activity is related to haemolysis extent, renal tissue damage or renal disease as well hyperlactaemia. Enzymatic changes underscore its release from tissues in necrosis or increased synthesis. LDH was determined in plasma using the Architect c8000 Abbott Diagnostic Clinical Chemistry Analyser (Abbott Laboratories, Illinois, USA).

\subsubsection{AA5-Pectin patch Influence Aldosterone (ALD) and Arginine Vasopressin (AVP)}

Levels of ALD and AVP are affected in malaria and were estimated to show the influence of AA administration on renal electrolyte handling. Malaria increases levels of both hormones through sodium wasting and volume depletion for ALD and AVP, respectively. Blood samples collected from treated and untreated groups of SD rats were used in ELISA methods hormonal estimates. ALD estimations: An Elabscience ALD (Aldosterone) ELISA kit Catalog No, EEL-0070 (Elabscience Biotechnology Co. Ltd., WuHan, P. R. C.) was used to measure the hormone concentration following manufacturer's instructions. The detection limit was $15.63-1000 \mathrm{pg} / \mathrm{mL}$ with a sensitivity of $9.38 \mathrm{pg} / \mathrm{mL}$. AVP estimations: An Elabscience Rat ADH (Antidiuretic Hormone) ELISA kit Catalog No, E-EL-0552 (Elabscience Biotechnology Co. Ltd., WuHan, P. R. C.) was used to measure the hormone concentration following manufacturer's instructions. The detection limit was $31.25-2000 \mathrm{pg} / \mathrm{mL}$ with a sensitivity of $18.75 \mathrm{pg} / \mathrm{mL}$.

\subsubsection{Influence of AA on Oxidative Stress (OS) in Malaria}

OS infringes upon normal electrolytes handling in the proximal and distal convoluted tubules. The activities of superoxide dismutase (SOD), glutathione peroxidase (GPx) and levels of malonyl aldehyde (MDA) were measured in kidney tissues to estimate influence of AA on OS. SOD activity estimation: Tissue SOD activities of kidney homogenates from NIC, AA treated and IC SD rats were assessed by measuring the dismutation of superoxide radicals generated by xanthine oxidase and hypoxanthine in a convenient 96 well format using a commercially available from abcam Superoxide Dismutase Activity Assay kit 
(colorimetric) catalogue no. ab65354 (abcam, Canada) according the manufacturer's instructions. GPx activity estimation: Activity of GPx was determined in AA-pectin patch applied and control animals' kidney tissues using abcam Glutathione Peroxidase Activity Assay kit (colorimetric) catalogue no. ab102530 (abcam, Canada) according the manufacturer's instructions. MDA concentration estimation: MDA levels in kidney tissues from NIC, IC and infected-AA treated animals were estimated using a previously described method [14] with some modifications.

\subsection{Statistical Analysis}

Data are presented as the means \pm standard error of mean (SEM). Overall statistical comparisons between the control means and experimental groups were performed with GraphPad InStat Software (version 5.00, GraphPad Prism software (San Diego, California, USA), using one-way analysis of variance (ANOVA), followed by Tukey-Kramer post hoc multiple comparison test. A value of $p<0.05$ was considered significant.

\section{Results}

\subsection{Influence of AA5-Pectin Patch on\% Parasitaemia}

The degree of $\%$ parasitaemia decrease indicated the efficacy treatment on malaria. A comparisons of $\%$ parasitaemia during the sub-chronic study was made between AA5-pectin applied animals and controls (Table 1). AA5TDDS administration had lower\% parasitaemia at days 7-12 compared to IC and CHQ treatment $(\mathrm{p}<0.05$, respectively) and at day 21 compared to CHQ treatment.

Table 1.\% Parasitaemia comparisons between AA5-TDDS (AA 5mg/kg transdermal drug delivery system) application and controls. Values are presented as means $\pm S E M(n=6$ per group). *, \# $p<0.05$ compared to the IC (infected not treated control) and $30 C H Q$ patch, respectively.

\begin{tabular}{|c|c|c|c|c|}
\hline \multirow[b]{2}{*}{ Protocol } & \multirow[b]{2}{*}{ Parameter } & \multicolumn{3}{|c|}{ Sub-chronic Study Time-Course } \\
\hline & & $\begin{array}{l}\text { Pre-patent (D3) \% } \\
\text { Parasitaemia }\end{array}$ & $\begin{array}{l}\text { Patent/Treatment (D7- } \\
10) \% \text { Parasitaemia }\end{array}$ & $\begin{array}{l}\text { Post-treatment (D21) \% } \\
\text { Parasitaemia }\end{array}$ \\
\hline $\begin{array}{l}\text { Post- infection Asiatic acid } \\
\text { Transdermal Drug Delivery }\end{array}$ & $\begin{array}{l}\text { IC } \\
\text { 30CHQ } \\
\text { AA5-TDDS }\end{array}$ & $\begin{array}{l}3.5 \pm 0.2 \\
2.17 \pm 0.3 \\
3.23 \pm 0.4\end{array}$ & $\begin{array}{l}13.00-69.33 \pm 8.6 \\
10.33-43.33 \pm 11.5 \\
17.42-27.68 \pm 5.7^{*} \#\end{array}$ & $\begin{array}{l}\mathrm{N} / \mathrm{A} \\
0.3 \pm 0.23 \\
0.00 \#\end{array}$ \\
\hline
\end{tabular}

\subsection{Influence of AA5-Pectin Patch on Physicochemical Changes}

Eating and drinking habits indicated the bio-physiochemical status of both infected and non-infected animals. Comparison of food and water intake together with \% weight gain was made between AA5-pectin and controls (Table 2). Physiochemical properties were significantly preserved by AA5-pectin patch compared to the IC, and CHQ ( $<0.05$, respectively).

Table 2. AA5-TDDS (AA 5mg/kg transdermal drug delivery system) influence on \% weight changes, food and water intake. Compared to controls values are represented as means \pm SEM. *, \# p<0.05compared to the NIC (non-infected control), IC (infected not treated control) and CHQ (30mg/kg chloroquine), respectively.

\begin{tabular}{|c|c|c|c|c|c|}
\hline Parameter & Protocol & & Pre-patent (D 3) & Patent/Treatment (D7-10) & Post-treatment (D 21) \\
\hline \multirow{4}{*}{$\begin{array}{l}\text { A. Food intake } \\
(\mathrm{g} / 100 \mathrm{~g})\end{array}$} & \multirow{4}{*}{$\begin{array}{l}\text { Post- infection } \\
\text { AA TDD treated }\end{array}$} & NIC & $11 \pm 1$ & $10 \pm 2$ & $12 \pm 2$ \\
\hline & & IC & $11 \pm 1$ & $6 \pm 4$ & N/A \\
\hline & & 30CHQ patch & $11 \pm 2$ & $9 \pm 1$ & $8 \pm 1$ \\
\hline & & AA5-TDDS & $11 \pm 1$ & $10 \pm 2 * \#$ & $12 \pm 2 \#$ \\
\hline \multirow{3}{*}{$\begin{array}{l}\text { B. Water intake } \\
(\mathrm{mL} / 100 \mathrm{~g})\end{array}$} & \multirow{3}{*}{$\begin{array}{l}\text { Post- infection } \\
\text { AA TDD treated }\end{array}$} & NIC & $15 \pm 1$ & $13 \pm 2$ & $15 \pm 2$ \\
\hline & & IC & $14 \pm 1$ & $7 \pm 2$ & N/A \\
\hline & & 30CHQ patch & $15 \pm 1$ & $13 \pm 1$ & $14 \pm 3$ \\
\hline \multirow{4}{*}{$\begin{array}{l}\text { C. } \% \text { body weight } \\
\text { change }\end{array}$} & \multirow{4}{*}{$\begin{array}{l}\text { Post- infection } \\
\text { AA TDD treated }\end{array}$} & NIC & $8 \pm 1$ & $12 \pm 1$ & $14 \pm 1$ \\
\hline & & IC & $8 \pm 4$ & $-4 \pm 2$ & N/A \\
\hline & & 30CHQ patch & $8 \pm 1$ & $10 \pm 1$ & $12 \pm 1$ \\
\hline & & AA5-TDDS & $8 \pm 1$ & $11+1 * \#$ & $15 \pm 1 \#$ \\
\hline
\end{tabular}

\subsection{Influence of AA5-Pectin Patch on Lactate Dehydrogenase (LDH) Activity}

The degree of haemolysis was reflected by the activity of LDH in plasma. The influence of AA5-pectin hydrogel matrix patch on LDH activity was compared (Table 3). Application of the AA5-pectin patch decreased LDH activity significantly on day 12 as compared to either IC or 30CHQ patch $(\mathrm{p}<0.05$, respectively).
Table 3. Influence of AA5-TDDS (AA $5 \mathrm{mg} / \mathrm{kg}$ transdermal drug delivery system) on lactate dehydrogenase activity compared to controls. Results presented as mean \pm SEM ( $n=6$ in each group). *, \# $p<0.05$ compared to IC (infected not treated control) and $30 \mathrm{CHQ}$ (30mg/kg chloroquine) -pectin patch, respectively.

\begin{tabular}{ll}
\hline Parameter & $\begin{array}{l}\text { Transdermal Delivery Post Treatment } \\
\text { Day 12LDH (U/L) }\end{array}$ \\
\hline NIC & $551.00 \pm 42.29$ \\
IC & $2453.00 \pm 235.20$ \\
CHQ 30mg/kg Patch & $1063.00 \pm 51.85$ \\
AA $5 \mathrm{mg} / \mathrm{kg} \mathrm{Patch}$ & $598.00 \pm 46.59 * \#$ \\
\hline
\end{tabular}




\subsection{Influence of AA5-Pectin Patch on Glomerular Filtration Rate [GFR]}

Changes of GFR overtime reflected the status of renal function in the NIC, IC and treated animals (Figure 1). Onceoff AA-pectin patch influence on GFR compared to NIC, IC and 30CHQ. AA5-TDDS application preserved GFR compared to IC and 30-patch treatment $(\mathrm{p}<0.05$, respectively) at pertinent time periods.

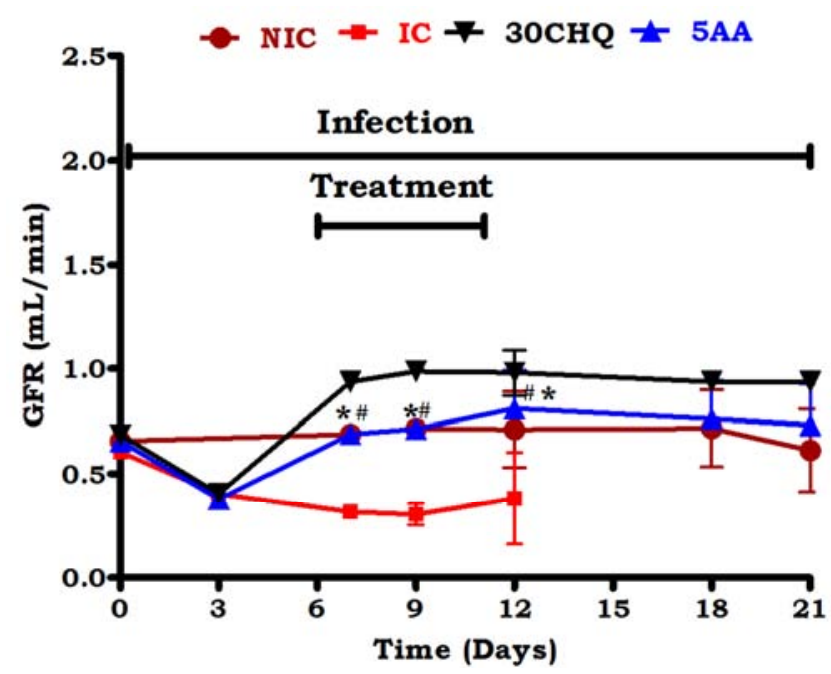

Figure 1. Comparison of GFR (Glomerular filtration rate) changes in AA5TDDS (AA 5mg/kg transdermal drug delivery system) and controls. Values are presented as means and vertical bars indicate $S E M(n=6$ per group). *, \# $p<0.05$ compared to IC (infected not treated control), NIC (non-infected control), 30CHQ (30mg/kg chloroquine) pectin matrix patch, respectively.

\subsection{Influence of AA5-Pectin Patch on Urine $\mathrm{Na}^{+}$Output (Mmol/L/Day)}

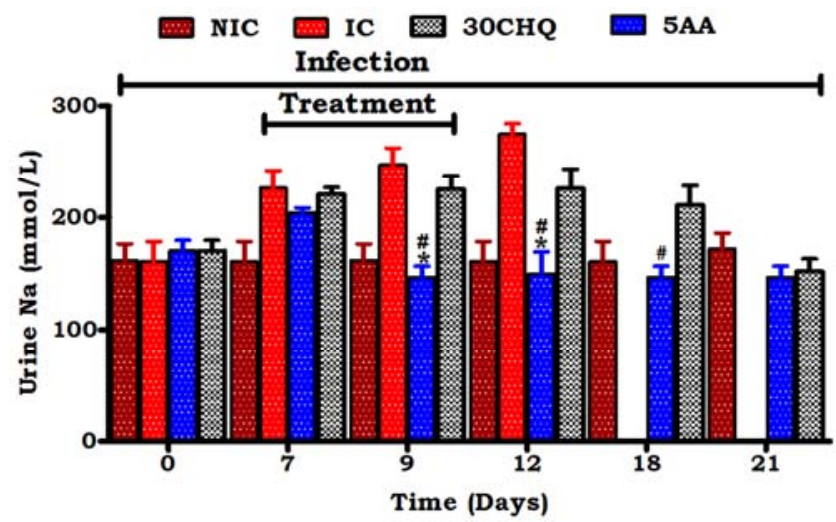

Figure 2. Urine $\mathrm{Na}^{+}$of $\mathrm{AA5}-\mathrm{TDDS}$ (AA $5 \mathrm{mg} / \mathrm{kg}$ transdermal drug delivery system) applied animals compared to controls. Results presented as mean \pm SEM ( $n=6$ in each group). *, \#p<0.05 compared to IC (infected not treated control), 30CHQ (30mg/kg chloroquine)-pectin patch, respectively.

Renal $\mathrm{Na}^{+}$preservation was shown by the amount of $\mathrm{Na}^{+}$ in the urine. Comparison of urine $\mathrm{Na}^{+}$output in AA5-pectin patch applied animals and controls showed (Figure 2). AA5 patch to significantly preserved $\mathrm{Na}^{+}$excretion compared to IC and 30CHQ patch $(\mathrm{p}<0.05$, respectively) at all relevant times. Influence of AA5-pectin patch on $\mathrm{K}^{+}$output
(mmol/L/day): Renal handling of $\mathrm{K}^{+}$was shown by the urine $\mathrm{K}^{+}$activity, and urine $\mathrm{K}^{+}$output in AA5-pectin patch applied animals and controls (Figure 3 ). AA5 patch significantly preserved $\mathrm{K}^{+}$excretion compared to IC and 30CHQ patch $(\mathrm{p}<0.05$, respectively) at all relevant times

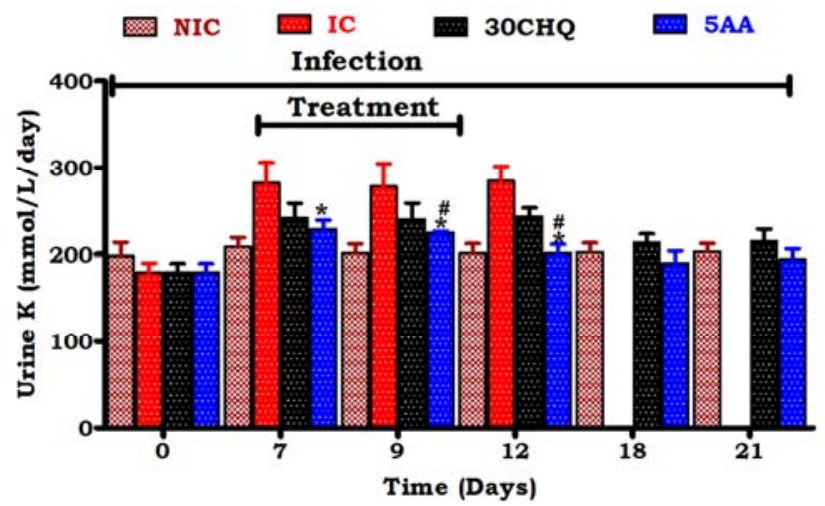

Figure 3. Urine $K^{+}$output for AA5-TDDS (AA $5 \mathrm{mg} / \mathrm{kg}$ transdermal drug delivery system) compared to controls. Values are presented as means and vertical bars indicate SEM ( $n=6$ per group). *, \#P<0.05 compared to IC (infected not treated control) and 30CHQ (30mg/kg chloroquine), respectively.

\subsection{Influence of AA5-Pectin Patch on Urine Cl Output (Mmol/L/Day)}

Urine $\mathrm{Cl}^{-}$output in AA5-pectin patch applied animals and controls (Figure 5). AA5 patch reduced $\mathrm{Cl}^{-}$excretion compared to IC and 30CHQ patch ( $\mathrm{p}<0.05$, respectively) at all relevant times. Influence of AA5-pectin patch on absolute $\mathrm{Na}^{+}$ excretion $(\mu \mathrm{mol} / \mathrm{mL} / \mathrm{min})$ : The influence of AA5-pectin patch on absolute $\mathrm{Na}^{+}$excretion and the controls were compared (Figure 6). Absolute $\mathrm{Na}^{+}$excretion was significantly lower in the AA5-pectin matrix patch administered infected animals compared to the IC and the 30CHQ-pectin matrix patch treated groups ( $\mathrm{p}<0.05$, respectively).

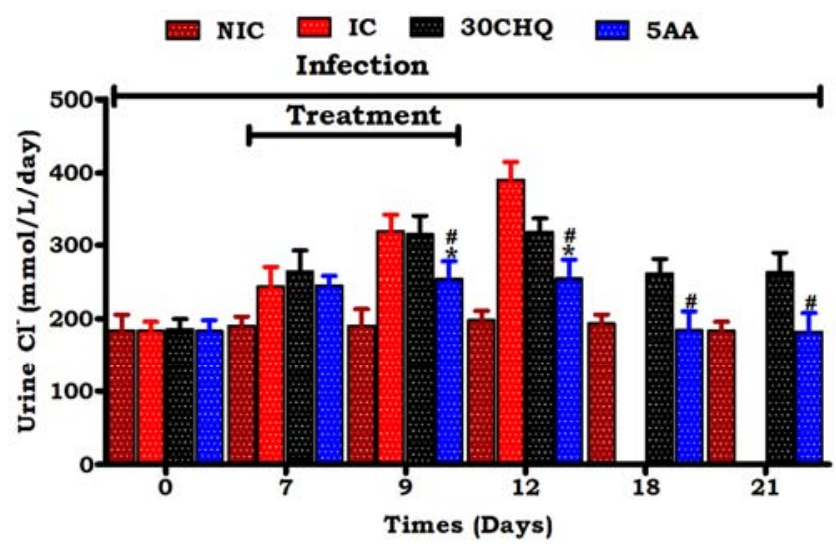

Figure 4. Urine $C l$ output of AA5-TDDS (AA $5 \mathrm{mg} / \mathrm{kg}$ transdermal drug delivery system) compared to controls. Results presented as mean $\pm S E M(n$ $=6$ in each group). *, \# $p<0.05$ compared to IC (infected not treated control), $30 \mathrm{CHQ}(30 \mathrm{mg} / \mathrm{kg}$ chloroquine)-pectin patch, respectively. 


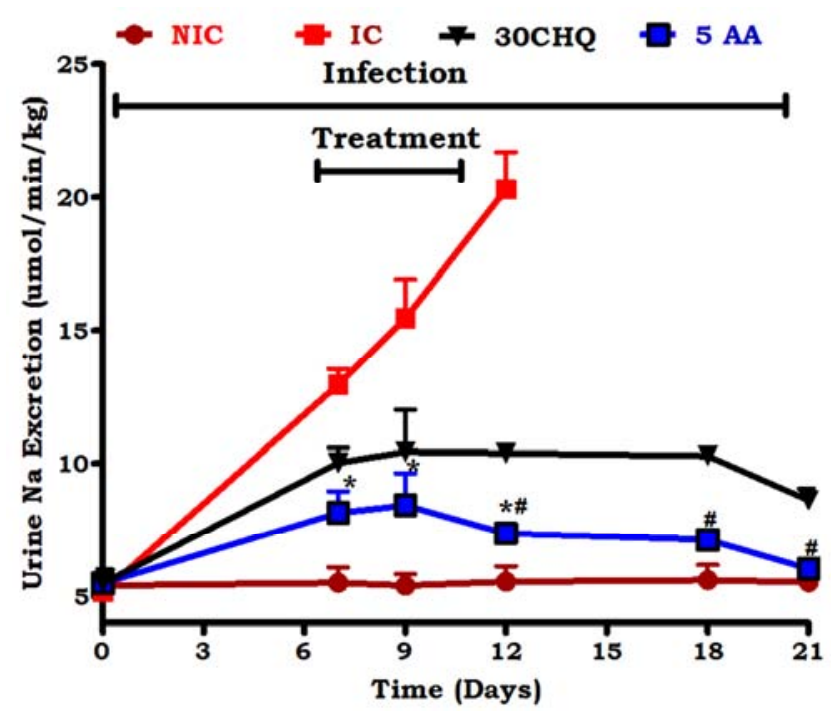

Figure 5. AA5-TDDS (AA $5 \mathrm{mg} / \mathrm{kg}$ transdermal drug delivery system) influence on absolute $\mathrm{Na}^{+}$excretion compared to controls. Results presented as mean $\pm S E M(n=6$ in each group). *, \# $p<0.05$ compared to IC (infected not treated control), $30 \mathrm{CHQ}$ (30mg/kg chloroquine)-pectin patch, respectively.

\subsection{Influence of AA5-Pectin Patch on Aldosterone}

Hormonal renal modulation of electrolyte handling was indicated by concentrations of ALD. A comparison of the influence of AA-pectin patch on ALD with controls was (Figure 7). Application of AA5-pectin patch significantly reduced ALD $(\mathrm{p}<0.05$, compared to IC and CHQ, respectively).

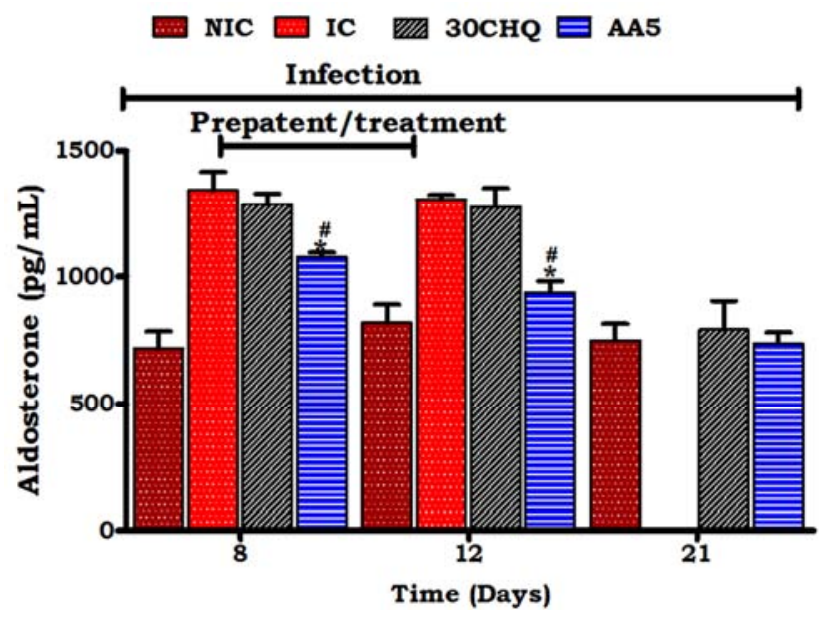

Figure 6. A comparison of AA5-TDDS (AA $5 \mathrm{mg} / \mathrm{kg}$ transdermal drug delivery system) influence on ALD against controls. Results presented as mean \pm SEM ( $n=6$ per group). *, \# $p<0.05$ compared to NIC (non-infected control), IC (infected not treated control) and CHQ (30mg/kg chloroquine), respectively.

\subsection{Influence of AA5-Pectin Patch on AVP Secretion}

Dehydration status and water balance was reflected by AVP concentrations in plasma. The influence of AA-pectin patch application is compared to controls (Figure7). Once-off AA-pectin patch application suppressed AVP significantly compared to both the IC and CHQ patch $(\mathrm{p}<0.05$, respectively). Influence of AA5-pectin patch on superoxide dismutase (SOD): Oxidative status of the animals administered AA, CHQ and IC groups was indicated by SOD concentration in tissues of the kidney. The influence of AA5pectin patch application on SOD activity is compared to control animals (Table 4 [A]). Compared to IC and CHQ, AA5-pectin patch administration did significantly increase enzyme activity $(p<0.05)$ in kidney. Influence of AA5-pectin patch on glutathione peroxidase (GPx): Oxidative status of the animals administered AA, CHQ and IC groups was indicated by GPx concentration in tissues of the kidney. AA5-pectin patch influence on GPx was compared to control animal groups in (Table 4 [B]). Influence of AA5-pectin patch on malonyldialdehyde (MDA): The level of lipid peroxidation, a marker of oxidative stress in malaria, was reflected by amount of MDA in the kidney tissues. The levels of MDA in AA-pectin patch applied animals was compared to controls (Table $4[\mathrm{C}]$ ). AA5-pectin patch significantly reduced levels of MDA in kidney compared to IC $(\mathrm{p}<0.05)$.

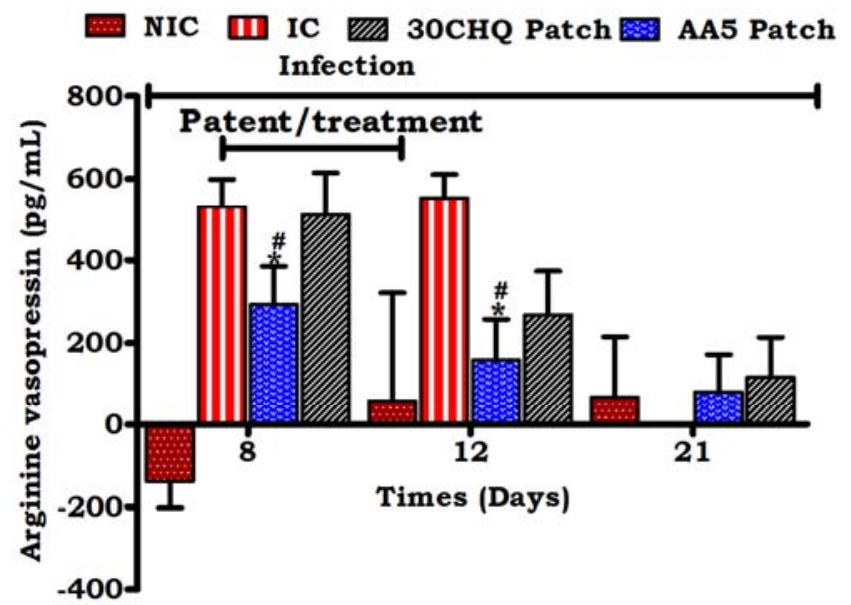

Figure 7. Influence of AA5-TDDS (AA $5 \mathrm{mg} / \mathrm{kg}$ transdermal drug delivery system) application compared with controls. Results presented as mean \pm SEM ( $n=6$ per group). *, \# $p<0.05$ compared to NIC (non-infected control), IC (infected not treated control) and CHQ (30mg/kg chloroquine), respectively.

Table 4. AA5-pectin patch effects on antioxidant status compared to controls. Superoxide dismutase (SOD), GPx and malonyldialdehyde (MDA) were determined. Values are presented as means \pm SEM ( $n=$ per group). *, \# $p<0.05$ compared to the NIC (non-infected control), IC (infected not treated control) and $\mathrm{CHQ}(30 \mathrm{mg} / \mathrm{kg}$ chloroquine), respectively.

\begin{tabular}{lll}
\hline \multirow{2}{*}{ Measured parameter } & Experimental group & Tissue source/organ \\
\cline { 2 - 3 } & & Kidney \\
\hline \multirow{3}{*}[\mathrm{A}]{$\mathrm{SOD}$ activity } & NIC & $26.34 \pm 13.6$ \\
$(\mathrm{mU} / \mathrm{mL})$ & IC & $2.58 \pm 2.9$ \\
& CHQ $30 \mathrm{mg} / \mathrm{kg}$ & $12.11 \pm 3.7$ \\
& AA5-TDDS $\mathrm{mg} / \mathrm{kg}$ & $23.34 \pm 4.1 * \#$ \\
& NIC & $56.67 \pm 2.9$ \\
{$[\mathrm{~B}] \mathrm{GPx}$ Activity } & IC & $8.23 \pm 2.6$ \\
$(\mathrm{mU} / \mathrm{mL})$ & CHQ $30 \mathrm{~m} / \mathrm{kg}$ & $44.87 \pm 2.5$ \\
& AA5-TDDS $\mathrm{mg} / \mathrm{kg}$ & $55.67 \pm 4.7 * \#$ \\
& NIC & $18.98 \pm 3.5$ \\
{$[\mathrm{C}] \mathrm{MDA}(\mathrm{nmol} / \mathrm{g}$} & IC & $49.78 \pm 5.8$ \\
protein) & CHQ 30mg/kg & $22.76 \pm 6.8$ \\
& AA5-TDDS $\mathrm{mg} / \mathrm{kg}$ & $20.12 \pm 7.7 *$ \\
\hline
\end{tabular}




\section{Discussion}

The systemic pathophysiology of malaria acute renal failure requires a wholesome approach bed-rocked on antimalarial agents with multiple anti-inflammatory properties, divergent from parasite load-reduction approaches [24]. We, therefore, report here for the very first time, the influence of transdermal drug delivery system of Asiatic acid on renal function and electrolyte handling in $P$. bergheiinfected SD male rats. This approach qualifies to be regarded as an anti-disease paradigm.

The combination of amphiphilic Asiatic acid and transdermal drug delivery system provide a robust framework for combating malaria providing a revolutionary way in which malaria may be managed. Asiatic acid-hydrogel matrix application provides a once-off treatment for malaria, in its own way, a drastic divergence from the known convoluted regimens of current antimalarial drugs in both dose, dosage frequency and administration route. The theoretic concentration delivered into the systemic circulation was several amplitudes lower than either oral or transdermal drug delivery system chloroquine at $3 \mu \mathrm{g} / \mathrm{kg}(6.14 \mathrm{nmol} / \mathrm{L})$ giving credence to the efficacy of Asiatic acid $5 \mathrm{mg} / \mathrm{kg}$-transdermal drug delivery system malaria suppression. In our laboratory, malaria posology is designed for a five day twice daily treatment course but Asiatic acid-transdermal drug delivery system reduced treatment to a once-off-three-day treatment protocol. This prototype reduced animal manipulation frequency, stress and conferred improved food and water intake as well as \% weight gain. Continued feed observed during infection is tacit indication of the animal overcoming the sickness behaviour which is characterised by food and water intake aversion, anorexia, cachexia and death.

Post-infection application of the patch preserved the animal wellbeing in general with profound effect of renal function and electrolyte handling. Indeed, malaria and malnutrition from inadequate food intake co-exist as factors contributing to high mortality in children [15]. An anorexic exposition in the infected non-treated animals (absent from Asiatic acid-transdermal drug delivery system treated animals) was typified by a negative $\%$ weight gain by day 12 making it necessary to humanely dispose those affected animals.

The preserved bio-physicochemical characteristic of animals on Asiatic acid $5 \mathrm{mg} / \mathrm{kg}$-pectin patch resulted in the improved glomerular filtration rate compared to either chloroquine $30 \mathrm{mg} / \mathrm{kg}$-patch or the infected non-treated animals. The influence of Asiatic acid on renal function is only limited to a report on Centella asiatica having diuretic effect cited by Singh et al (2010) from an untraceable source [44], making this current report the first to show improved glomerular filtration rate from Asiatic acid-transdermal drug delivery system administration. Moreover, the anti-parasitic efficacy of Asiatic acid has been reported recently in pre- and post-infection oral administration of Asiatic acid $(10 \mathrm{mg} / \mathrm{kg})$ to animal experiment $[21,22]$. Treatment with chloroquine patch resulted in a higher glomerular filtration rate during the treatment period as has been reported that chloroquine inhibits arginine vasopressin (antidiuretic hormone) which will invariably increase urine output and glomerular filtration rate [29] leading to hypovolaemia, dehydration and increased arginine vasopressin concentration in return. Observations have also been made that oral chloroquine administration has more pronounced effects on glomerular filtration rate than transdermal drug delivery system chloroquine [28] and Asiatic acid-transdermal drug delivery system seem to have the opposite effect. Furthermore, Patel et al (2015), using silico structure-based virtual screening approach have shown Asiatic acid to be non-nephrotoxic [36] which effect has been corroborated by current findings that Asiatic acidtransdermal drug delivery system had protective effect in malaria shown by the recovery and preservation of glomerular filtration rate.

The influence of Asiatic acid-transdermal drug delivery system application on urine $\mathrm{Na}^{+}$output was significantly lower compared to the infected non-treated control and chloroquine-patch treatment. However, there was an increase in urine $\mathrm{Na}^{+}$output on day 7 , at the initiation of treatment, which was reflected by a non-significant inflection in the glomerular filtration rate when compared to the non-infected treated control during the pertinent time point which we could not explain based on these results. We, however, speculate as an interpretation, that the observed urine $\mathrm{Na}^{+}$ output rise could have been a parasite induced effect as this trend was similar to the infected non-treated control urine $\mathrm{Na}^{+}$output and glomerular filtration rate. The initial diffusion, for lack of knowledge on the Asiatic acid transportation mechanism through the dermis at present, into the systemic circulation may have coincided with an increase in parasitaemia causing a fall in glomerular filtration rate and subsequently a rise in urine $\mathrm{Na}^{+}$output. A cursory inspection of the \% parasitaemia will show that indeed, there was a higher transition in parasitaemia between pre-patent (day 3) and the patent/treatment (day 7) periods in the order of $14.19 \%>9.5 \%>8.16 \%$ for Asiatic acid, infected non-treated control and chloroquine, respectively. It may also be instructive to mention that a single urine $\mathrm{Na}^{+}$output is more an indication of the concentration of the urine and its volume, in the absence of the urine osmolality and may not be reflective of incipient kidneys disease. Our observations were the higher the urine $\mathrm{Na}^{+}$the less urine volume excreted and the more concentrated the urine was. The homeostasis of urine outflow, by convention, depends on a variety of factors to include water intake. We were able to demonstrate that the Asiatic acid-pectin patch applied animals did preserve their water intake for the duration of the study. However, a constantly higher and rising urine $\mathrm{Na}^{+}$may have indicated kidney disease as we observed with the infected non-treated control and chloroquine-patch groups.

Other researcher have shown that malaria induces increased $\mathrm{Na}^{+}$excretion through the induction of nitric oxide (NO) $[10,41]$. Inducible nitric oxide synthesis (iNOS) is upregulated in malaria generating excessive NO, a soluble gas synthesized from L-arginine, which inhibits $\mathrm{Na}^{+} / \mathrm{K}^{+}$ ATPase pump responsible for the active reabsorption of the 
ion in the proximal convoluted tubules and other ells. When combined with oxygen, $\mathrm{NO}$ forms peroxinite $\left(\mathrm{ONOO}^{-}\right)$a highly oxidative compound which might be responsible for inhibition of the $\mathrm{Na}^{+}$transporters.

Asiatic acid has been suggested to inhibit iNOS, cyclooxygenase- 2 , interleukin- 6 , interleukin- $1 \beta$ and tumour necrosis factor- $\alpha$ expression by downregulating nuclear factor-kappa beta via $\mathrm{Ik} \beta$ kinase and mitogen-activated protein kinase [12]. These inflammatory mediators are invariably upregulated in malaria. The anti-inflammatory effect of Asiatic acid has been demonstrated in other studies. There is a higher probability that the preservation of $\mathrm{Na}^{+}$ excretion could be through the Asiatic acid-transdermal drug delivery system administration induced protection of the $\mathrm{Na}^{+} / \mathrm{K}^{+}$ATPase from oxidative inhibition. The infected nontreated control group had significantly higher $\mathrm{Na}^{+}$excretion. On the other hand, chloroquine-patch high $\mathrm{Na}^{+}$urine excretion during patent/treatment period and beyond was expected as from previous studies. Other researchers have reported that chloroquine increases renal $\mathrm{Na}^{+}$excretion by inhibiting AVP or ADH [30, 31]. Synergistic effects of the parasite and chloroquine in $\mathrm{Na}^{+}$depletion may be involved in the hyponatraemia of malaria and may persist after successful disease treatment [47].

The absolute urine $\mathrm{Na}^{+}$excretion was observed to be continuously rising in the infected non-treated controls showing a sodium wasting process through transporters inhibition. We cannot currently explain why the parasite causes $\mathrm{Na}^{+}$depletion, weakening its own survival chances with the demise of the host. We speculate that an excessive $\mathrm{Na}^{+}$diuresis is not an intended outcome by the parasite. A sequelae from host over-responsiveness to infection through immune reactivity, oxidative stress and inflammation might also be at play. These aspects were resolved by the application of Asiatic acid-amidated pectin patch in our experiments as compared to infected non-treated control and chloroquine. This may suggest that application of Asiatic acid-transdermal drug delivery system may have an antidisease effect on renal $\mathrm{Na}^{+}$handling through restoration of the $\mathrm{Na}^{+} / \mathrm{K}^{+}$ATPase pump or preservation of other mechanism affected by malaria infection.

The urine $\mathrm{K}^{+}$output was significantly elevated in the untreated as well as the chloroquine patch treated animal groups when compared to the Asiatic acid-pectin patch treated animals. This was possibly a reflection of the hyperkalaemia occurring as result of the ATPase pump failure to maintain $\mathrm{Na}^{+}$within the extracellular compartment as well as increased haemolysis. The ATPase pump does not affect renal electrolyte handling only but most cell membranes including red blood cells (RBC's) with subsequent $\mathrm{K}^{+}$movement into the extracellular space.

The wider ramification of inflammation driven NO excess tends to affect RBC's metabolism and membrane integrity and deformability which results in RBC's (both parasitizedpRBC's and non-parasitized-npRBC's) removal by the spleen [19] with resultant severe malaria anaemia [8]. We did not observe a rise in urine $\mathrm{K}^{+}$output in the AA5-pectin patch applied animals in contrast to the infected non-treated animals and the chloroquine-patch treated animals. This may indicate a reduction or absence of haemolysis which may be traced back to the anti-inflammatory effect of Asiatic acid on RBC membrane integrity. The antioxidant capacity of AA could also have quenched ROS generation while the prooxidant capacity may have eradicated the parasite to preserve the kidney function.

To corroborate the renal electrolyte preservation capacity of AA5-pectin patch was the estimation of urine $\mathrm{Cl}^{-}$output. Urine $\mathrm{Cl}^{-}$output was elevated in the $\mathrm{IC}$ as well as the chloroquine-patch treated animals, but remained significantly correlated to the non-infected treated animals' values except during treatment. Noteworthy is the fact that, application of the Asiatic acid-pectin patch was commenced when the parasitaemia had already reached patent and stable state and the reversal of the electrolyte dysregulation observed for $\mathrm{Cl}^{-}$ but not observed in the infected non-treated animals urine $\mathrm{Cl}^{-}$ output indicated the influence of Asiatic acid on renal electrolyte handling. An increase in urine $\mathrm{Cl}^{-}$output, besides depicting a concentrated urine excretion, may also be an indicator of acid base balance disturbances. In nonrespiratory acidosis there is a depletion of $\mathrm{HCO}_{3}^{-}$and an increase in $\mathrm{H}^{+}$which necessitates conversation of the latter in the proximal convoluted tubules. To maintain an electrolyte neutrality, $\mathrm{Cl}^{-}$shifts from the tubular cells into the lumen in exchange and preservation of $\mathrm{HCO}_{3}{ }^{-}$. The hydronium ion is also excreted in exchange for $\mathrm{K}^{+}$or $\mathrm{Na}^{+}$but due the ATPase pump failure in malaria, this may not happen. As a result non-respiratory acidosis escalates worsening the compromised renal function.

Besides impairment of $\mathrm{Na}^{+} / \mathrm{K}^{+}$ATPase pump by increased oxidative stress, ATP rundown seen in malaria through Poly (ADP-ribose) polymerase activation by $\mathrm{ONOO}^{-}$in malaria may also contribute to pump failure with the same results. Besides inhibition of $\mathrm{Na}^{+} / \mathrm{K}^{+}$ATPase pump, NO also inhibits endothelial $\mathrm{Na}^{+}$channels $(\mathrm{ENaC})$. Other electrolyte transporters such as the $\mathrm{Na}^{+} / \mathrm{H}^{+}$exchanger that facilitate proton excretion and $\mathrm{HCO}_{3}{ }^{-}$reabsorption depend on the $\mathrm{Na}^{+}$ electrochemical gradient created by the ATPase. Inhibition of the pump may, therefore, result in acidosis [5]. However, Asiatic acid has been reported to alleviate haemodynamic and metabolic aberrations in rats with metabolic syndrome via the equilibration of endothelial nitric oxide synthase (eNOS) and induced nitric oxide synthase expression together with oxidative stress and inflammation reduction [35]. These reports are in support of our findings that Asiatic acid-pectin patch application suppressed parasitaemia as well as the abolition of other malaria pathophysiology.

Due to the inhibition of $\mathrm{ENaC}$, aldosterone is unable to effect $\mathrm{Na}^{+}$retention resulting in salt-losing nephropathy characterised by dehydration and non-respiratory acidosis and a concomitant rise in the hormone's plasma concentration. When aldosterone activity is elevated, in rising natriuresis, a condition termed pseudohypoaldosteronism type 1 results in congenital conditions characterised by $\mathrm{ENaC}$ failure [2]. We observed 
the same scenario during the initiation of AA-TDDS application on day 8. Significantly higher activity of aldosterone corresponded to raised urine $\mathrm{Na}^{+}$output and a downward transitory inflection in the glomerular filtration rate which could indicate hypovolaemia from reduced food and water intake, increased RBC's destruction or both. This may also imply that before therapeutic dose of Asiatic acid was reached, there might have been inhibition of the $\mathrm{ENaC}$ resulting in hyperaldosteronism. Reactivation of the channel, through possible quenching of oxidative stress or oxygen reactive species or both might have normalized $\mathrm{Na}^{+}$ excretion. In stark contrast to Asiatic acid-pectin patch effect was the infected non-treated and chloroquine-patch treated controls showing raised aldosterone and urine $\mathrm{Na}^{+}$ concentrations, indications of possible excessive ATPase pump inhibition.

AVP activity for Asiatic acid-pectin patch applied animals, during the day 8 time-point, was higher than for other timepoints triggered by possible hypovolaemia and increasing osmolality. However, the activity was comparatively lower than both the infected non-treated and chloroquine-patch treated groups. Indeed, chloroquine has been shown to influence increased $\mathrm{Na}^{+}$urine excretion [31] through inhibition of cyclic AMP (cAMP) or via generation of NO [1]. Both mechanism cause an increase in plasma vasopressin as we observed in these experiments. The resultant diuresis and natriuresis are hall marks of chloroquine treatment which we were corrected in Asiatic acid-transdermal drug delivery system application. In contrast to this finding, $C$. asiatica extract has been reported to increase cAMP content in tissues [46]. Asiatic acid could have used the same mechanism in preservation of renal electrolyte handling.

To probe further the influence of Asiatic acid-transdermal drug delivery system on oxidative stress, we measured the activity of antioxidant enzymes and the level of peroxidation in the kidney. Significantly higher superoxide dismutase activity was observed in the animals on Asiatic acid-pectin patch. Also, significantly raised was glutathione peroxidase from Asiatic acid- transdermal drug delivery system pathed animals as compared to both the infected non-treated and the chloroquine-patch treated animals. On the other hand animals applied the Asiatic acid-TDDS patch had lower MDA compared to the chloroquine-patch and the infected nontreated groups. Others workers have also described similar findings with oral administration of Asiatic acid at higher doses, than reported here, in non-malaria conditions [12]. The antioxidant role of Asiatic acid-pectin patch in the preservation of renal electrolyte handling may have been through reactivation of the ATPase pump as well as hormonal influence of water metabolism showing possible anti-disease effects of Asiatic acid on kidney function. Further work, however, may be needed to explore the possible interaction of Asiatic acid with electrolyte channels and transporters, the interaction of Asiatic acid with malaria toxins that elicit inflammatory reactions and the general metabolic effects of the amphiphilic nature of Asiatic acid in malaria.

\section{Conclusion}

It has been able to demonstrated that AA5-amidated pectin hydrogel matrix patch application reduced the dosing frequency, dosage and the duration of treatment. Also shown was the ability of Asiatic acid-pectin patch application to preserve food and water intake as well as weight gain and suppress parasitaemia. Asiatic acidtransdermal drug delivery system ameliorated the disease aspect of malaria through increasing antioxidant capacity, reduction in renal lipid peroxidation, preservation of renal function and electrolyte homeostasis together with maintenance of renal hormonal function. In addition, it was observed that pseudohypoaldosteronism was a reversible condition in malaria with proper treatment as a slight increase in urine $\mathrm{Na}^{+}$output was attenuated by Asiatic acid-transdermal drug delivery system application. Speculation is made in this research that the antioxidant capacity of Asiatic acid-transdermal drug delivery system through quenching of $\mathrm{NO}, \mathrm{ROS}, \mathrm{ONOO}^{-}$seem to influence renal function and electrolyte handling through preservation of the $\mathrm{Na}^{+} / \mathrm{K}^{+}$ATPase pump and ENaC. These findings warrant possible application of Asiatic acid-transdermal drug delivery system as antimalarial therapy in the future.

\section{Acknowledgements}

Honour goes posthumously, to Professor Cephas Tagumirwa Musabayane the dearly beloved, departed supervisor and mentor of this work. Professor Fanie R Van Heerden is highly honoured for the initial AA that allowed this project to start. The Discipline of Physiology Endocrinology Group, Mr. M. Luvuno in particular, is highly appreciated for the tremendous contributions in animal and laboratory experiments. Special mention is herein made of the contributions of Ms Sibiya HP and Mbatha B whose animal handling techniques whetted the author's skills in this field. This study was made possible through University of KwaZulu Natal, College of Health Sciences (CHS) scholarship granted (CHS 213574054) obtained by the first author (GAM).

\section{Conflict of Interests}

All the authors do not have any possible conflicts of interest.

\section{Abbreviations}

AA-asiatic acid, TDDS-transdermal drug delivery system, AVP-arginine vasopressin, ADH-antidiuretic hormone, CACentella asiatica, pRBC's-parasitized red blood cells, npRBC's-non-parasitized red blood cells 


\section{References}

[1] Ahmed M H and Osman M M. (2007) Why does chloroquine impair renal function? Chloroquine may modulate the ranal tubular response to vasopressin either directly by inhibiting cyclic AMP generation, or indirectly via nitric oxide. Med Hypothesis. 68 (1): 140-143.10.1016/j.mehy.2006.06.012.

[2] Amin N, Alvi N S, Barth J H, Field H P, Finlay E, Tyerman K, et al. (2013) Pseudohypoaldosteronism type 1: clinical features and management in infancy. Endocrine Diabet Metab case report. 13: 0010.10.1530/EDM-13-0010.

[3] Barsoum R S. (2000) Malarial aute renal failure. J Am Soc Nephrol 11: 2147-2154.

[4] Changa K-H and Stevenson M M. (2004) Malarial anaemia: mechanisms and implications of insufficient erythropoiesis during blood-stage malaria. Internat J Parasitol 34: 15011516.

[5] Clark I A and Cowden W B. (2003) The pathophysiology of falciparum malaria. Pharmacol Therapeut 99: 221-260.

[6] Feasey N A, Dougan G, Kingsley R A, Heyderman R S, and Gordon M A. (2012) Invasive non-typhoidal salmonella disease: an emerging and neglected tropical disease in Africa. Lancet 363. 79: 2489-2499.10.1016/S0140-6736 (11) 617522.

[7] Forbes J M, Coughlan M T and Cooper M E. (2008) Oxidative Stress as a Major Culprit in Kidney Disease in Diabetes. Diabetes 57 (6): 1446-1454.http://dx.doi.org/10.2337/db080057.

[8] Ghosh K and Ghosh K. (2007) Pathogenesis of anemia in malaria: a concise review. Parasitol Res 101: 14631469.10.1007/s00436-007-0742-1.

[9] Gumede B, Folbb P and Ryffela B. (2003) Oral artesunate prevents Plasmodium berghei Anka infection in mice. Parasitol Internation. 52: 53-59.

[10] Guzman N J, Fang M Z, Tang S S, Ingelfinger J R, and Garg L C. (1995) Autocrine inhibition of $\mathrm{Na}+/ \mathrm{K}+$-ATPase by nitric oxide in mouse proximal tubule epithelial cells. J Clin Invest 95: 2083-2088.

[11] Hanson J, Hossain A, Charunwatthana P, Hassan M U, Davis T M E, Lam S W K, et al. (2009) Hyponatremia in Severe Malaria: Evidence for an Appropriate Anti-diuretic Hormone Response to Hypovolemia. Am J Trop Med Hyg 80 (1): 141145 .

[12] Huang S-S, Chiu C-S, Chen H-J, Hou W-C, Sheu M-J, Lin Y$C$, et al. (2011) Antinociceptive Activities and the Mechanisms of Anti-Inflammation of Asiatic Acid in Mice. Evid-Based Complemen Altern Med. 2011: 10 pages. 10.1155/2011/895857.

[13] Karney W W and Tong M J. (1972) Malabsorption in Plasmodium falciparum malaria. Am J Trop Med Hyg 21: 1-5.

[14] Kasapoglu M and Özben T. (2001) Alterations of antioxidant enzymes and oxidative stress markers in aging. Experiment Gerontol 36: 209-220.

[15] Kateera F, Ingabire C H, Hakizimana E, Kalinda P, Mens P F, Grobusch M P, et al. (2015) Malaria, anaemia and under- nutrition: three frequently co-existing conditions among preschool children in rural Rwanda. Malaria $\mathrm{J}$ 14: 440.10.1186/s12936-015-0973-z.

[16] Khan F Y. (2009) An imported case of P. falciparum malaria presenting as black water fever with acute renal failure.. Travel Med Infect Dis.; 7: 378-380.

[17] Khan R, Quaiser S and Haque S F. (2013) Malarial acute kidney injury: Prognostic markers. Ann Trop Med Public Health 6: 280-284.

[18] Locatelli F, Canaud B, Eckardt K-U, Stenvinkel P, Wanner C, and Zoccali C. (2003) Oxidative stress in end-stage renal disease: an emerging threat to patient outcome. Nephrol Dial Transplant. 18: 1272-1280.10.1093/ndt/gfg074.

[19] Maitland K and Marsh K. (2004) Pathophysiology of severe malaria in children. Acta Tropica 90 131-140.

[20] Mavondo G A, Mkhwananzi B N and Mabandla M V. (2016) Pre-infection administration of asiatic acid retards parasitaemia induction in Plasmodium berghei murine malaria infected Sprague Dawley rats. Malar J. 15: 226.10.1186/s12936-016-1278-6.

[21] Mavondo G A, Mkhwanazi B N and Mabandla M V. (2016) Pre-infection administration of Asiatic acid retards parasitaemia induction in $P$. berghei murine malaria infected Sprague-Dawley rats Malar J. 15: 226.

[22] Mavondo G A, Mkhwanazi B N, Mabandla M V, and Musabayane C T. (2016) Asiatic acid influences parasitaemia reduction and ameliorate malaria anaemia in $P$. berghei infected Sprague Dawley male rats. BMC CAM. Inprint.

[23] Mavondo G A and Musabauane C T. (2016) Asiatic acidpectin hydrogel matrix patch transdermal delivery system influences parasitaemia suppression and inflammation reduction in $P$. berghei murine malaria infected SpragueDawley rats. APJTM 9: 11721180.http://dx.doi.org/10.1016/j.apjtm.2016.10.008.

[24] Miller L H, Ackerman H C, Su X-z, and Wellems T E. (2013) Malaria biology and disease pathogenesis: insights for new treatments. Nature Med. 19 (2): 156-167.

[25] Moxon C A, Wassmer S C, Milner D A J, Chisala N V, Taylor T E, and Seydel K B. (2013) Loss of endothelial protein C receptors links coagulation and inflammation to parasite sequestration in cerebral malaria in African children. Blood. 122 (5): 842-851.

[26] Murambiwa P, Tufts M, Mukaratirwa S, van Heerden F R, and Musabayane C T. (2013) Evaluation of efficacy of transdermal delivery of chloroquine on Plasmodium berghei-infected male Sprague-Dawley rats and effects on blood glucose and renal electrolyte handling. Endocrine Abstracts 13: P203.10.1530/endoabs.31. P203.

[27] Musabayane C T, Munjeri O and Matavire T P. (2003) Transdermal delivery of chloroquine by amidated pectin hydrogel matrix patch in the rats. Renal failure. 25: 525-534.

[28] Musabayane C T, Munjeri O P and Matavire B. (2003) Transdermal Delivery of Chloroquine by Amidated Pectin Hydrogel Matrix Patch in the Rat. Renal failure 25: 525-553.

[29] Musabayane C T, Ndhlovu C E, Mamutse G, Bwititi P, and Balment R J. (1993) Acute chloroquine administration increases renal sodium excretion J Tropic Med Hyg 96: 305310. 
[30] Musabayane C T, Wargent E T and Balment R J. (2000) Chloroquine inhibits arginine vasopressin production in isolated rat inner medullary segments induced cAMP collecting duct. Renal Failure. 22: 27-37.

[31] Musabayane C T, Windle R J, Forsling M L, and Balment R J. (1996) Arginine vasopressin mediates the chloroquine induced increase in renal sodium excretion,. Tropic Med Internat Health 1: 542-550.

[32] Naicker S, Aboud O and Gharbi M B. (2008) Epidemiology of acute kidney injury in Africa. Semin Nephrol. 28 348353.10.1016/j.semnephrol.2008.04.003.

[33] Naqvi R, Ahmad E, Akhtar F, Naqvi A, and Rizvi A. (2003) Outcome in severe acute renal failure associate with malaria. Nephrol Dial Transplant. 18: 1820-1823.

[34] Olsson R A and Johnston E H. (1969) Histopathologic changes and small-bowel absorption in falciparum malaria. Am J Trop Med Hyg 18: 355-359.

[35] Pakdeechote P, Bunbupha S, Kukongviriyapan U, Prachaney P, Chrisanapant W, and Kukongviriyapan V. (2014) Asiatic Acid Alleviates Hemodynamic and Metabolic Alterations via Restoring eNOS/iNOS Expression, Oxidative Stress, and Inflammation in Diet-Induced Metabolic Syndrome Rats. Nutrients 6 (1): 355-370.10.3390/nu6010355.

[36] Patel H, Dhangar K, Sonawane Y, Surana S, Karpoormath R, Thapliyal N, et al. (2015) In search of selective 11 beta-HSD type 1 inhibitors without nephrotoxicity: An approach to resolve the metabolic syndrome by virtual based screening. Arabian J Chem 10.1016/j.arabjc.2015.08.003.

[37] Pino P, Vouldoukis I and Kolb J P. (2003) Plasmodium falciparum-infected erythrocyte adhesion induces caspase activation and apoptosis in human endothelial cells. J Infect Dis 187: 1283-1290.

[38] Plewes K, Haider H S, Kingston H W F, Yeo T W, Ghose A, Hossain A A, et al. (2015) Severe falciparum malaria treated with artesunate complicated by delayed onset haemolysis and acute kidney injury. Malar J 14: 246.10.1186/s12936-0150760-x.

[39] Prasad R N and Virk K J. (1993) Malaria as a cause of diarrhoea-a review. P N G Med J 36: 337-341.

[40] Reisinger E C, Fritzsche C, Krause R, and Krejs G J. (2005) Diarrhea caused by primarily non-gastrointestinal infections. Nat Clin Pract Gastroenterol Hepatol. 2: 216-222.

[41] Roczniak A and Burns K D. (1996) Nitric oxide stimulates guanylate cyclase and regulates sodium transport in rabbit proximal tubule. Am J Physiol 270: F106-F115.
[42] Rolling T, Agbenyega T, Issifou S, Adegnika A A, Sylverken J, and Spahlinger D e a. (2014) Delayed hemolysis after treatment with parenteral artesunate in African children with severe malaria-a double-center prospective study. J Infect Dis 209: 1921-1928.

[43] Salman I M, Sattar M A, Abdullah N A, Ameer O Z, Basri B, Hussain N M, et al. (2010) Renal functional \& haemodynamic changes following acute unilateral renal denervation in Sprague Dawley rats. Indian J Med Res 131: 76-82.

[44] Singh S, Gautam A, Sharma A, and Batra A. (2010) Centella asiatica (L): a plant with immense medicinal potential but threatened. Intern J Pharceut Scie Rev Res. 4 (2): 9-12.

[45] Taniguchi T, Miyauchi E, Nakamura S, Hirai M, Suzue K, Imai T, et al. (2015) Plasmodium berghei ANKA causes intestinal malaria associated with dysbiosis. Scientific Reports 5 (Article number: 15699).

[46] Tholon I, Neliat G, Chesne C, Saboureau D, Perrier E, and Branka J E. (2002) An in vitro, ex vivo, and in vivo demonstration of the lipolytic effect of slimming liposomes: An unexpected $\alpha$ (2)-adrenergic antagonism. J Cosmet Sci. 6: 209-218.

[47] van Wolfswinkel M E, Hesselink D A, Zietse R, Hoorn E J, and vanGenderen P J. (2010) Hyponatraemia in imported malaria is common and associated with disease severity. Malar J. 9: 140.10.1186/1475-2875-9-140.

[48] WHO. (1990) Severe and complicated malaria. World Health Organization, Division of Control of Tropical Diseases. Trans R Soc Trop Med Hyg 84 (Suppl 2): 1-65.

[49] Wilairatana P, Meddings J B, Ho M, Vannaphan S, and Looareesuwan S. (1997) Increased gastrointestinal permeability in patients with Plasmodium falciparum malaria. Clin Infect Dis 24: 430-435.

[50] XU M F, XIONG Y Y, LIU J K, QIAN J J, Zhu L, and GAO J. (2012) Asiatic acid, a pentacyclic triterpene in Centella asiatica, attenuates glutamate-induced cognitive deficits in mice and apoptosis in SH-SY5Y cells. Acta Pharmacol Sinic. 33: $578-587$.

[51] Yan S-L, Yang H-T, Lee Y-L, Lin C-C, Chang M-H, and Yin M-C. (2014) Asiatic Acid Ameliorates Hepatic Lipid Accumulation and Insulin Resistance in Mice Consuming a High-Fat Diet. J Agric Food Chem 62 4625-4631.10.1021/jf501165z.

[52] Zhang J, Lisha A I, Tingting L V, Jiang X, and Liu F. (2013) Asiatic acid, a triterpene, inhibits cell proliferation through regulating the expression of focal adhesion kinase in multiple myeloma cells. Oncol letters. 6: 17621766.10.3892/ol.2013.1597. 